\title{
TRABAJO COLABORATIVO EN LA ASIGNATURA DOCUMENTACIÓN INFORMATIVA: UNA PROPUESTA DIDÁCTICA PARA CONOCER EL PROCESO DOCUMENTAL EN LOS MEDIOS
}

\author{
Natalia Meléndez-Malavé1: Universidad de Málaga. España \\ natalia@uma.es
}

\section{RESUMEN}

A fin de que el alumnado de la asignatura Documentación Informativa adquiriera un conocimiento de primera mano acerca de cómo se gestionan las diferentes tareas de la Cadena Documental (selección, análisis, almacenamiento, búsqueda y difusión) en centros de documentación de empresas informativas, se diseñó un ejercicio basado en una selección de textos procedentes de publicaciones especializadas. En ellos, los propios responsables de varias unidades de archivo de diferentes medios explican paso a paso la organización de su departamento. La actividad consistía, pues, en extraer y exponer a partir de dichos textos una serie de datos sobre cada una de las fases arriba indicadas, previa división de la clase en pequeños grupos interdependientes, cuyo intercambio de información conduce a mejorar tanto el aprendizaje individual como el de todo el colectivo. El presente texto describe esta experiencia de aplicación de la técnica didáctica del aprendizaje cooperativo (AC) en estudiantes de la Facultad de Ciencias de la Comunicación de la Universidad de Málaga de la asignatura Documentación Informativa, materia troncal de $2^{\circ}$ curso de la Titulación de Periodismo. En los resultados de la investigación se interpretan las encuestas realizadas al alumnado al final de la actividad.

PALABRAS CLAVE: Comunicación - Periodismo - Documentación Informativa Aprendizaje cooperativo - Aprendizaje colaborativo.

\footnotetext{
${ }^{1}$ Autor correspondiente

Natalia Meléndez-Malavé: Profesora ayudante doctora. Universidad de Málaga (España)

Correo: natalia@uma.es
} 


\title{
COLLABORATIVE WORK IN THE INFORMATIVE DOCUMENTATION COURSE : A DIDACTIC PROPOSAL FOR THE LEARNING OF DOCUMENTARY PROCESS IN THE MEDIA
}

\begin{abstract}
Intending to make the students of the subject Informative Documentation to acquire a first-hand knowledge about how to manage the different tasks of the Documentary Process (acquisition, analysis, storage, search, and recall) in mass media-related documentation centres, an exercise based in a selection of texts from specialist journals was designed. In this journals, the people in charge of some media companies file services, spell out their department's management. The exercise basically consisted in extract and present, using those texts as a basis, a series of records about every one of the previous phases, after dividing the class in small, interdependents groups. This exchange of information led to improve the individual learning as well as the group one. This text describes this didactic tool of cooperative learning experience in Universidad de Málaga students of the subject Informative Documentation, main subject in the second year of Journalism Degree in the Faculty of Communication Science. In the research results, the polls fulfilled by the students at the end of the exercise are evaluated.
\end{abstract}

KEY WORDS: Communication - Journalism - Informative Documentation Cooperative Learning - Collaborative Learning.

\section{INTRODUCCIÓN}

La propuesta que aquí se presenta parte de la base de una necesaria adaptación de la asignatura Documentación Informativa a los nuevos planteamientos proclamados por el Espacio Europeo de Educación Superior que, entre otros aspectos, fomenta una mayor interactividad entre profesorado y alumnado, convirtiendo a estos últimos en el centro del proceso de aprendizaje. De hecho, ya se viene reclamando hace un tiempo la conveniencia de alejarse de una orientación excesivamente teórica de la materia "con un escaso componente didáctico, y por lo tanto poco adaptada a los nuevos tiempos", como bien señala Nicolás Bas Martín (Bas Martín, 2006, p. 113) en un artículo en el que se analizan las necesidades de renovación de las prácticas docentes en Documentación Informativa. 
En dicho texto se recuerda, además, que el nuevo marco en el que nos sitúa el llamado Plan Bolonia supone el diseño de nuevas estrategias en las que se favorezca que los estudiantes "aprendan haciendo", y de ahí surge la actividad de trabajo colaborativo que aquí recogemos. Así pues, nos proponemos abordar de manera descriptiva y evaluar los resultados de una experiencia de aplicación de la técnica didáctica del aprendizaje cooperativo (AC) en esta asignatura de Documentación para futuros informadores y más concretamente en uno de los contenidos centrales del temario, el proceso o cadena documental.

En la elección de esta herramienta se tomaron en cuenta investigaciones previas que han valorado los resultados de la aplicación de AC y que han señalado entre sus beneficios un aumento de la motivación por aprender, una mayor retención del conocimiento, una comprensión más profunda, y una actitud más positiva hacia la asignatura (Felder \& Brent, 1996).

Para comprender los criterios con los que se trabaja en el AC se ha de tener en cuenta que una de las teorías más influyentes en este tipo de aprendizaje se centra en la interdependencia social. Así, Johnson, Johnson y Holubec (1999) hacen especial hincapié en el concepto de interdependencia positiva, de tal modo que el éxito personal no es posible sin el de todos los demás, de modo que un individuo se ve favorecido por la aportación del grupo. En efecto, al hablar de AC prevalece la idea de que los estudiantes pueden obtener mejores resultados trabajando juntos y en pequeños grupos que haciéndolo independientemente, de tal forma que "el resultado de un trabajo hecho en un grupo colaborativo tienen un valor superior al que tendría la suma de los trabajos individuales de cada miembro de dicho grupo", (Bernaza Rodríguez \& Lee Tenorio, 2005, p. 15).

En este punto juzgamos necesario detenernos brevemente e introducir una reflexión acerca de los conceptos de aprendizaje cooperativo y colaborativo, a veces usados indistintamente, a veces diferenciados. A pesar del sutil matiz indicado por Panitz (2001) -según el cual el aprendizaje cooperativo supondría un mayor control por parte del docente frente al aprendizaje colaborativo donde sería el alumnado quien dirigiría las interacciones y decisiones sobre su aprendizaje-, en nuestro caso hemos empleado bibliografía referida tanto a un término como a otro. Sea cual sea la expresión empleada, en definitiva, el objetivo es alejarse de la unidireccionalidad de la clase magistral a favor de una fórmula caracterizada por la participación del alumnado, que debe indagar para extraer sus propias conclusiones, siempre en interacción con el resto del grupo, de manera que ejerce un rol mucho más activo en su aprendizaje. 


\section{METODOLOGÍA}

\subsection{Objetivos}

La actividad surge en el marco de la investigación-acción en el aula, al detectar la docente encargada de la asignatura a través de las pruebas de evaluación que no se terminaban de alcanzar en parte del alumnado algunos de los objetivos principales de la asignatura, a saber: un conocimiento más próximo a la realidad del funcionamiento de los centros de documentación en los medios de comunicación tanto escritos como audiovisuales, así como de la aplicación en estos de las distintas fases de la cadena documental (selección, análisis, almacenamiento, búsqueda y difusión). Así pues, se planteó la posibilidad de sustituir la explicación de estos contenidos en clase magistral, como se había venido haciendo en cursos precedentes, por una experiencia de $\mathrm{AC}$, en la que los propios estudiantes elaboraran de un modo más personal su aprendizaje, compartiendo el fruto de esta asimilación con los compañeros.

La finalidad principal es, por tanto, mejorar la adquisición de conocimientos de un contenido muy concreto de la asignatura. Pero este objetivo general se completa además con otros más específicos, relacionados a su vez con las necesidades pedagógicas que implica la convergencia europea:

a) Fomentar competencias tales como las habilidades comunicativas, de trabajo autónomo, en equipo y de análisis y síntesis.

b) Acercar al alumnado a su futuro entorno profesional, a través de la familiarización con situaciones reales.

c) Mejorar el manejo de fuentes especializadas.

d) Hacer las clases más dinámicas y participativas, fomentando la implicación del alumnado.

e) Potenciar el compromiso y los comportamientos solidarios y éticos.

\subsection{Descripción de la experiencia}

La actividad propuesta fue desarrollada en concreto por estudiantes de la Facultad de Ciencias de la Comunicación de la Universidad de Málaga de dos grupos de la asignatura Documentación Informativa, materia troncal de $2^{\circ}$ curso de la Licenciatura de Periodismo, de cuya docencia es responsable la autora. Los estudiantes tienen nociones previas, explicadas a lo largo del curso, acerca de todo el proceso que experimentan los documentos que entran a formar parte de un archivo de un medio de comunicación. 
Pero, como se ha adelantado, a fin de que el alumnado percibiera como algo menos "abstracto" todo lo relacionado con la organización en los departamentos de documentación de los medios -con los que, como profesionales, deberán estar familiarizados en un futuro- se diseñó un ejercicio basado en una selección de textos procedentes de publicaciones especializadas en Documentación.

En ellos, investigadores expertos o los propios responsables de varios centros documentales de emisoras de radio, cadenas de televisión y periódicos con especial hincapié en los archivos fotográficos- explican paso a paso en qué consisten sus funciones y cómo se gestionan las diferentes tareas de la cadena documental agrupadas en las sucesivas fases de selección, análisis, almacenamiento, búsqueda y difusión, antes indicadas. La actividad consistía, pues, en extraer y exponer a partir de dichos textos - un total de seis, dos por cada medio- una serie de datos sobre cada una de estas fases, previa división de la clase en seis pequeños grupos interdependientes, cuyo intercambio de información conduce a mejorar tanto el aprendizaje individual como el de todo el colectivo.

La organización del trabajo en clase comienza con la formación de los grupos, que se realiza de manera aleatoria, utilizando un sencillo sistema basado, con alguna transformación, en la técnica Philips 66: a cada seis estudiantes se les asigna un número del 1 al 6 y después todos los que tienen el mismo número deben buscarse y colocarse juntos. En este caso concreto, a los grupos 1 y 2 se les repartieron los textos sobre prensa, a los 3 y 4 sobre radio, y la televisión les correspondió a los equipos 5 y 6.

Los centros documentales a analizar correspondían a medios de comunicación variados, tanto públicos como privados, tanto nacionales como autonómicos. La selección no es difícil de realizar haciendo búsquedas en publicaciones como El profesional de la Información o Hipertext.net, así como en algunos manuales de Documentación que dedican capítulos al funcionamiento de determinados servicios documentales en medios.

En todo caso, se considera recomendable hacer una revisión de la literatura cada vez que se vaya a poner en práctica el ejercicio para aportar información lo más actualizada posible. De cualquier forma, juzgamos que es una ventaja más de la propuesta el hecho de que suponga para los universitarios la lectura de textos procedentes de publicaciones académicas y revistas especializadas, algo fundamental en su formación y aún más en el caso de futuros comunicadores que deberán acostumbrarse al contacto con este tipo de fuentes y sus autores.

Por otra parte, el funcionamiento interno de cada uno de los seis grupos exige además una subdivisión para que cada cual se encargue de una tarea que luego repercuta en la resolución final de la actividad, tal y como sientan las bases del AC. 
Para ello, además del artículo correspondiente, se entrega una hoja de instrucciones a cada grupo en la que se les indica, entre otros aspectos, que en el seno de cada equipo el trabajo se dividirá a su vez por parejas o tríos (dependiendo del número de estudiantes en cada clase) que realizan las siguientes funciones:

a) Subgrupo "Selección": este subgrupo es el encargado de identificar en el texto datos tales como los criterios de selección de los fondos que alberga su centro documental, la cantidad, procedencia interna o externa, formatos y tipos de documentos que lo alimentan, la política de adquisición (compras, canjes, donaciones). En suma, qué documentos se deciden conservar y cuáles no.

b) Subgrupo "Análisis y Almacenamiento": Aquí se unifican estos dos aspectos centrales del proceso documentales y el equipo queda encargados de detectar en el texto, por un lado, lo referente al tratamiento o análisis al que se someten los documentos una vez seleccionados (tareas de descripción de los documentos como la catalogación, la indización, alusiones a lenguajes documentales como tesauros o descriptores, etc.). Y, por otro lado, en cuanto al almacenamiento o preservación, deberán especificar sobre todo si el texto habla de documentos conservados física o digitalmente y en qué formato, si se hace alguna referencia a las condiciones del espacio, al mobiliario, etc.

c) Subgrupo "Búsqueda, Recuperación y Difusión": Son los responsables de tratar lo concerniente a la última fase del proceso, la que realizan mayoritariamente los usuarios. Contempla aspectos como quiénes son esos posibles usuarios (si el servicio atiende también peticiones externas, por ejemplo), qué tipo de documentos se demandan y cómo se realizan esas solicitudes de información. Habrá que atender muy especialmente a las referencias a las bases de datos que emplean para sus consultas y los lenguajes documentales con los que comunican esas necesidades de búsqueda.

d) Subgrupo "Secretarios-Organizadores": Se ocupan de dos labores: primero, encontrar en el texto los datos más básicos para poder identificar y describir a sus compañeros de los otros equipos el centro de documentación que les ha correspondido. Por ejemplo, lo relativo a la organización y características del departamento (instalaciones, número de trabajadores y organigrama, algunos datos históricos si el archivo tiene cierta antigüedad, etc.). Y, segundo, sobre todo, unifican las tres partes anteriores proporcionadas por sus compañeros de grupo en un documento único que plasmará de manera esquemática cómo se aplica cada fase de la cadena documental en el centro de documentación que les ha correspondido. Los integrantes de este subgrupo son los responsables de que ese documento resultante sea enviado antes de una fecha límite por correo electrónico a la profesora para su revisión y puesta a disposición de toda la clase a través de la plataforma virtual de la asignatura. 
En cuanto a la secuenciación temporal, dependiendo de las horas de clase y cómo estén distribuidas, el trabajo puede tener que desarrollarse en varios días. Se comienza el día en que se explica la dinámica, se conforman los grupos y se reparten los recursos (texto y hoja de instrucciones).

El resto de la jornada o la clase siguiente se dedica a terminar la tarea, siempre bajo la supervisión docente: se ha de prestar atención al trabajo de los grupos y atender cualquier duda o problema que pueda surgir. Finalmente, la última fase de la actividad consistirá en realizar una puesta en común en la que los integrantes de cada grupo explican a sus compañeros brevemente cada parte que les ha correspondido analizar -hay que recordar que, aunque en la plataforma virtual contarán con todos los documentos, cada grupo ha trabajado en profundidad sólo su texto, y es necesario que se expliquen unos a otros los rasgos básicos del centro documental que les ha correspondido.

Así, bajo la dirección de la docente, que procura dinamizar el debate, durante esta fase de exposición se extraen conclusiones sobre semejanzas y diferencias entre centros documentales del mismo medio de comunicación (por ejemplo, si las dos emisoras de radio trabajan de igual manera o usan herramientas distintas) o entre medios distintos (por ejemplo, si hay rasgos comunes en un centro televisivo con respecto a uno de prensa). A tenor de nuestra experiencia, aparecerán temas interesantes como el valor patrimonial del material documental o la constante necesidad de renovación tecnológica, por ejemplo.

Como vemos, la interdependencia propia del AC supone trabajar con un engranaje en el que cada estudiante debe aplicar el máximo esmero e implicación por el bien de todos los compañeros, que deberán tener a su disposición todo los documentos unificados en los que se esquematizará cada fase de la cadena en cada uno de los seis departamentos de Documentación.

La propia mecánica del AC garantiza, salvo excepciones, el compromiso del alumnado pero, para reforzar dicho compromiso, el material resultante de esta tarea suele ser objeto de una de las preguntas en la prueba final de la asignatura. Normalmente, se trata de cuestiones que pueden contestarse simplemente con haber participado en la realización de la actividad y lo que interesa es comprobar si se ha interiorizado y comprendido por entero lo visto en el ejercicio, es decir, no se les fuerza a memorizar los seis documentos resultantes, algo que carecería de sentido.

Más allá de los resultados en el examen de la asignatura, se consideró fundamental realizar una evaluación de esta experiencia, para mejorarla de cara a cursos posteriores o cambiar de herramienta si fuese necesario. 
Por tanto, para comprobar si verdaderamente el alumnado se veía favorecido por esta innovación se realizaron encuestas al final de la actividad en las que se valoraban distintos aspectos: hasta 20 preguntas cerradas empleando la escala Likert y dos más de carácter abierto para introducir mejoras en el diseño de la actividad para su aplicación en futuros cursos.

\section{ANÁLISIS Y DISCUSIÓN}

\subsection{Resultados y evaluación}

La encuesta de evaluación de la actividad fue contestada por 77 estudiantes en total, procedentes del turno de mañana y del turno de tarde la asignatura. Los 20 ítems de respuesta cerrada de que constaba consistían en afirmaciones cuyo acuerdo o desacuerdo tenían que puntuar los estudiantes en una gradación que iba del 1 (totalmente en desacuerdo) al 5 (totalmente de acuerdo). Calificar con un 2 indicaba estar en desacuerdo y con un 4 , de acuerdo.

El intervalo intermedio (3) suponía estar indeciso. Para facilitar la comprensión y evitar confusiones se redactaron las proposiciones en positivo y se agruparon de tal manera que al comienzo y al cierre se encontraban las preguntas que versaban sobre la valoración general de la tarea, el grado de dificultad y otras cuestiones de la percepción del alumnado sobre su experiencia personal; otro grupo de preguntas indagaba en la idoneidad del material facilitado y el papel de la docente y las explicaciones para comprender los objetivos del ejercicio; también se preguntaba sobre la dinámica de trabajo en grupo, así como acerca de la fase de puesta en común; asimismo, se añadieron ítems sobre la asimilación de los contenidos del tema. Además se incluyó un espacio abierto en el que los estudiantes podían señalar tres aspectos que modificarían de la actividad y tres que no cambiarían.

Al ser preguntados por la valoración general de esta actividad la mitad de los estudiantes $(50,65 \%)$ se mostraron de acuerdo en considerarla como útil para su aprendizaje y además el $24,68 \%$ se mostró totalmente de acuerdo, no computándose ningún desacuerdo absoluto (1 en la escala del cuestionario), por lo que sumando estos resultados podemos considerar en principio exitosa la puesta en marcha de esta experiencia, a juicio del alumnado.

Continuando en el plano de los aspectos más positivos, entre los puntos con mayor aceptación de la tarea encontramos el hecho de que se realice presencialmente en el aula y en horario lectivo, ítem que concentra los mayores porcentajes de valor superior, con un $38,96 \%$ de total acuerdo y un 36,36\% de acuerdo "simple", es decir consignando el valor 4 . 
También como satisfactorio situamos el hecho de que más de la mitad (54,55\%) consideraban la actividad como útil para mejorar sus conocimientos sobre el tema. En este sentido, el 46,75\% afirmaban comprender mejor cómo funciona un centro de documentación después de haber realizado la tarea, e idéntico porcentaje señalaba entender mejor la cadena documental, que ya anteriormente había sido objeto de atención en clase magistral en apartados anteriores del temario, y cuya comprensión se ve reforzada con la actividad, a tenor de las respuestas.

Destacamos también que el 32,47\% mostró consenso con la idea de que no le había resultado difícil desarrollar la actividad (a lo que podemos sumar un 24,68\% que declara que no le había resultado en absoluto dificultosa, siendo este ítem al que se asignó el mayor índice de valor 1 de toda la encuesta).

Al ser interrogados sobre si habían aprendido mejor de esta manera que con una clase magistral, las respuestas son también óptimas, ya que más de la mitad otorgan los valores superiores, con el $27,27 \%$ de acuerdo y el 31,17\% totalmente de acuerdo con esta aseveración.

Los recursos facilitados también son mayoritariamente bien aceptados, un 44,16\% está de acuerdo en la afirmación de que la hoja de instrucciones es comprensible y, aún más, un 48,05\% opina lo mismo de los artículos de dónde debían extraer la información requerida e incluso en las respuestas abiertas se destaca la selección de textos como un aspecto que debe permanecer. Este es uno de los puntos que consideramos más favorables en el diseño de la tarea, pues al estar basada en artículos académicos nos aseguramos de que el alumnado tome contacto con la literatura especializada a la que por experiencia sabemos que tan difícil es en ocasiones acercarles.

Por otro lado, no cabe duda de que el desempeño de esta propuesta exige un esfuerzo por parte del profesorado, algo de lo que fueron conscientes los estudiantes al otorgar el máximo valor a la satisfacción con el papel ejercido por la docente en un 50,65\% de los casos. No obstante, existen aún varias cuestiones en las que introducir mejoras, según los datos que arroja la encuesta de evaluación. Entre los aspectos con los que el alumnado se mostró más crítico encontramos: el trabajo en grupo y la puesta en común.

En el primer caso, los estudiantes no se muestran mayoritariamente satisfechos con el nivel de implicación de todos los integrantes de su grupo. Aquí el nivel de respaldo a la afirmación de que la participación de todos los componentes del grupo ha sido adecuada desciende a sólo un 25,94\%. En este punto observamos un desequilibrio entre la percepción del propio esfuerzo (por ejemplo, el 41,56\% cree que ha logrado los objetivos de la tarea) y la más negativa actitud hacia el trabajo del equipo: pocos respaldan la idea de que el trabajo en grupo contribuye a que se sientan más motivados e incluso hay un alto nivel de total desacuerdo con dicho enunciado, un 9.09\% adiudicó el valor 1 a este ítem. 
Es más, en las respuestas abiertas del cuestionario, entre las más numerosas peticiones de modificación encontramos tanto la propuesta de que los grupos sean más reducidos, como de que no se elijan sus integrantes al azar.

Paradójicamente, este tipo de formación de los equipos también se encuentra en las respuestas de lo que no se cambiaría, por lo que existe entre otra parte del alumnado una buena acogida al hecho de trabajar con compañeros distintos a los habituales, a lo que se añade que un $41,56 \%$ declara que ha mejorado su habilidad para interactuar en clase.

En todo caso, el trabajo en grupo siempre supone un aspecto controvertido: mientras que algunos piden control de asistencia o que el cumplimiento de la actividad suponga una nota extra, otros se muestran satisfechos con la realización grupal de la tarea y la forma de reparto del trabajo en diferentes roles.

Una división de opiniones parecida se da a la hora de valorar la puesta en común en clase de los resultados de cada grupo, ya que aunque buena parte $(45,45 \%)$ considera útil la realización de esta fase de la actividad, no tantos comparten que la exposición les ayudase a conocer mejor el trabajo del resto, con un alto nivel de valores intermedios (37,66\%). Esto se explica más ampliamente en las respuestas abiertas, donde algunos proponen sustituir esta fórmula más cercana al debate - aunque más estructurada y formal- por exposiciones en la tarima acompañadas de material gráfico de apoyo.

Asimismo, el nivel de atención de algunos compañeros cuando otros tienen la palabra es también motivo de descontento, algo que efectivamente hay que estar controlando en todo momento cuando se ponen en práctica estas herramientas docentes. Así pues, este resultado nos alerta al profesorado para que tengamos presente que a lo largo del desarrollo de este tipo de ejercicio de expresión oral hay que intentar evitar la disminución de atención y para que, como conductores de la dinámica, seamos firmes ante la falta del respeto debido al turno de palabra de cada estudiante. En todo caso, deducimos que la visión del resultado de la puesta en común es muy personal, según lo experimentado por cada estudiante, pues el número de ellos que cambiaría esta parte de la actividad es similar al de los que no lo modificarían.

\section{CONCLUSIONES}

La adopción del trabajo colaborativo como estrategia didáctica supone una mayor interacción en las clases, convirtiendo lo que podía ser una mera transmisión de contenidos de corte teórico en una actividad dinámica y participativa, en la que los estudiantes construyen su propio aprendizaje. 
A pesar de que supone un gran cambio con respecto al modo en que el alumnado estaba habituado a concebir las clases, el esfuerzo en todo caso, fructifica en unos resultados muy favorables, no sólo reflejados en la encuesta, sino también en la prueba de conocimientos y, sobre todo, en una serie de factores que se perciben en el transcurso de la tarea: una mayor implicación del alumnado, que no se limita a recibir datos para luego memorizar (y posiblemente olvidar pasado un tiempo) sino que experimenta con la autonomía para trabajar, con la creación de contenidos y a la vez se acerca a las prácticas reales de su futuro ámbito profesional.

Además, lo que consideramos muy importante, el estudiante ejercita la responsabilidad, toda vez que de la culminación de su parte de la tarea depende no sólo su éxito sino el del conjunto de su clase, uno de los rasgos más destacables del trabajo colaborativo. Por ello encontramos que resulta apropiado para su adopción como innovación educativa en estudios superiores tanto de Comunicación, donde es especialmente necesario potenciar el intercambio de información y la colaboración en un entorno de trabajo colectivo, como en otras disciplinas. No obstante, sería inmodesto confirmar concluyentemente con esta somera investigación que se mejoran capacidades tales como las comunicativas o de trabajo en equipo, entre otras, pues es algo que merecería un estudio amplio a o largo de todo el periodo formativo de nuestro alumnado.

Lo que sí estamos en condiciones de aportar a través de los resultados de la experiencia son orientaciones, toda vez que la actitud más responsable como docentes debe ser la de centrarnos en los aspectos a mejorar y en las recomendaciones que puedan resultar de utilidad para profesores que deseen aplicar esta técnica pedagógica y adaptarla a su asignatura. Asimismo, lo que sí podemos afirmar es que como mínimo se trabajan en clase un conjunto de objetivos y competencias de una manera más aproximada a lo que el nuevo marco nos exige, y esto en sí ya supone un primer avance, sobre todo frente al estático modelo en el que era el profesorado el centro del proceso de enseñanza-aprendizaje.

Cabe añadir para finalizar que, pese a la espontaneidad del trabajo presencial con el grupo, hay que ser conscientes de la ineludible estructuración que se exige tanto antes, como durante y después de la ejecución de la actividad. Para empezar, hay un trabajo previo de planificación tanto temporal como de preparación de los recursos y selección de los textos más adecuados.

Además, durante su puesta en práctica juzgamos fundamental el control del responsable de la docencia, quien deberá estar alerta durante el desarrollo de la tarea para que no se produzcan disfunciones en el trabajo en grupo o, como se ha puesto de manifiesto, en la ejecución de la fase expositiva. 
Del mismo modo, se deben revisar los documentos finales que se compartirán en la plataforma virtual al final de la experiencia. En cualquier caso, y a pesar de la existencia de facetas que mejorar, en rasgos generales, valoramos de manera positiva los resultados de adaptar esta fórmula innovadora para inculcar entre el alumnado los conocimientos y habilidades explicados más arriba.

\section{REFERENCIAS}

Aronson, E. (1978). The jigsaw classroom. Beverly Hills: CA Sage.

Ausubel, D., Novak, J. \& Hanesian, H. (1991). Psicología Educativa: un punto de vista cognoscitivo. México: Trillas.

Bas Martín, N. (2006). La documentación informativa en el nuevo espacio europeo de educación superior: Reflexiones desde la didáctica. Revista General de Información y Documentación, 16(2): 111-126.

Bernaza Rodríguez, G. \& Lee Tenorio, F. (2005). El aprendizaje colaborativo: una vía para la educación de postgrado. Revista Iberoamericana de Educación, 37(3).

Cohen, E. (1999). Complex Instruction: Equity in Cooperative Learning Classrooms. Theory into Practice, 38(2): 80-86.

Delgado Peña, J. J. (2008). El Aprendizaje Cooperativo en la Universidad ante el reto del EEES: Luces y sombras del cambio de paradigma en la enseñanza universitaria. Trabajo presentado al I Jornadas Andaluzas de Investigación e Innovación Educativa. Granada.

Delgado Peña, J. J. (2009). El Aprendizaje Cooperativo y Significativo en la programación docente dentro del ámbito del EEES. Trabajo presentado al III Jornadas de innovación educativa y enseñanza virtual de la Universidad de Málaga. Recuperado el 27 de mayo de 2011, de http://www.uma.es/ieducat/new_ieducat/ambito_1/Com.1PIE07.081.pdf.

Felder, R. M. \& Brent, R. (1996). Navigating the bumpy road to student-centered Instruction. College Teaching, 44: 43-47.

García, R., Traver, J. A. \& Candela, I. (2001). Aprendizaje cooperativo: fundamentos, características y técnicas. Madrid: Editorial CCS.

Johnson, D., Johnson, R. \& Holubec, E. J.(1999). El aprendizaje cooperativo. Barcelona: Paidós.

Ovejero, A. (1990). El aprendizaje cooperativo: una alternativa eficaz a la enseñanza tradicional. Barcelona: PPU. 
Panitz, T. (2001). Collaborative versus cooperative learning- a comparison of the two concepts which will help us understand the underlying nature of interactive learning. Recuperado el 12 de junio de 2011, de http://www.capecod.net/ tpanitz/tedspage/tedsarticles/coopdefinition.htm

Sharan, Y. \& Sharan, S. (2004): El desarrollo del aprendizaje cooperativo a través de la investigación en grupo. Sevilla: MCEP.

Sierra Sánchez, J. \& Sotelo González, J. (2010). Métodos De Innovación docente aplicados a los estudios de Ciencias de la Comunicación. Madrid: Fragua.

Sotelo, J. \& Marco, J. C. (2008). Una reflexión sobre la metodología docente sobre la enseñanza del Periodismo en el marco del EEES. En El nuevo perfil del profesor universitario en el EEES: claves para la renovación metodológica. Valladolid: Servicio de Publicaciones UEMC.

\section{Natalia Meléndez Malavé}

Doctora en Periodismo (2005) por la Universidad de Málaga (UMA). Licenciada en Periodismo (2000) por la UMA. Profesora ayudante doctora en el Departamento de Periodismo de la Facultad de Ciencias de la Comunicación de la UMA. Ha participado en numerosas publicaciones con los resultados de sus investigaciones, especializadas en dos líneas centrales: por un lado, formas y medios de comunicación no convencionales (humor gráfico, redes sociales) y sus conexiones con la representación, la identidad o los estereotipos y, por otro lado, innovación educativa en la enseñanza universitaria (aplicación de TIC, aprendizaje cooperativo, implantación del EEES). En este ámbito ha formado parte de diversos proyectos de investigación. 